\title{
A Novel Approach to Recycle Energy Using Piezoelectric Crystals
}

\author{
Arjun A.M., Ajay Sampath, Sandhya Thiyagarajan, and Arvind V
}

\begin{abstract}
- with the increasing demand for utilization of energy resources, there has been a deflation in the availability of resources and there have been many methods of recycling of energy resources. .It is our responsibility to ensure that we do not deplete all the energy resources leaving our future generations facing dangerous hazards. Our paper is one such attempt to reduce that burden. The method being employed by us is a new concept, which not only utilizes the principle of piezoelectricity to harvest energy but it also employs pyroelectric crystals and microcontroller to create a kind of feedback, with a battery getting charged automatically ,till it becomes fully charged. With piezo electric crystals it is possible to harvest energy from vibrating structures.Pyroelectric effect occurs when change in temperature causes flow of charges. These two effects are used to charge the various components being used in this experiment while remaining energy is being used to charge the battery. Our model aims to create a cyclic flow of energy, thus ensuring very less or no wastage of energy.
\end{abstract}

Index terms-Microcontroller, piezoelectricity, pyroelectric effect.

\section{INTRODUCTION}

A variety of environmental problems now affect our entire world. Apparently, many people are comforted by the fact that they can afford to waste. Fig 1 indicates some of the primary energy sources in the year 2006 in India. Also the emissions of pollutants and greenhouse gases from fossil fuel-based electricity generation produce harmful gases. Most large scale thermoelectric power stations consume considerable amount of water for cooling purpose and boiling water. Hence by the process of recycling of energy, the greenhouse gas emissions in industrial plants are minimized. Recycling non-biodegradable waste rather than burning it, will contribute a lot to help reduce air pollution and greenhouse gases that depletes the ozone layer.

\section{OVERVIEW}

\section{A. Recycling of Energy}

Energy recycling is the energy recovery process of utilizing energy that would normally be wasted, by converting it into electric energy or thermal energy. There are various forms of recycling of energy which have existed so

Manuscript received October 11, 2011, revised October 17, 2011

Arjun A.M., Ajay Sampath and Arvind V are with Sri Venkateswara College of Engineering, Pennalur, Sriperumbudur, India (e-mail: arjun.champ@gmail.com; funchaparvind@gmail.com). shaa309@gmail.com;

Sandhya $\mathrm{T}$ is with Meenakshi Sundararajan Engineering College, Kodambakkam, Chennai, India (e-mail: sanshrey90@gmail.com). far like:-

Solar cells

Turbines

Reciprocating engines

Photovoltaic panels

Electro chemical conversion

Piezoelectricity

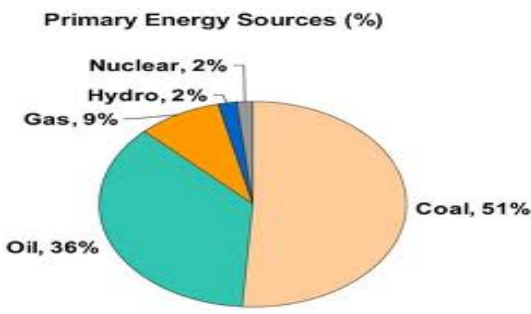

Fig. 1. Primary energy sources in India 2006

A major concern with the solar cell is that it is not cheap and it does not generate much power. The turbines have tedious installation process. Our paper introduces a new concept of energy recycling using the piezoelectric crystal and a microcontroller. This method is best utilized for efficient recycling of energy if it is put to proper use.

\section{B. Existing Projects}

There are quiet a lot of current projects involving piezo electricity as their main concept. Some of them are:

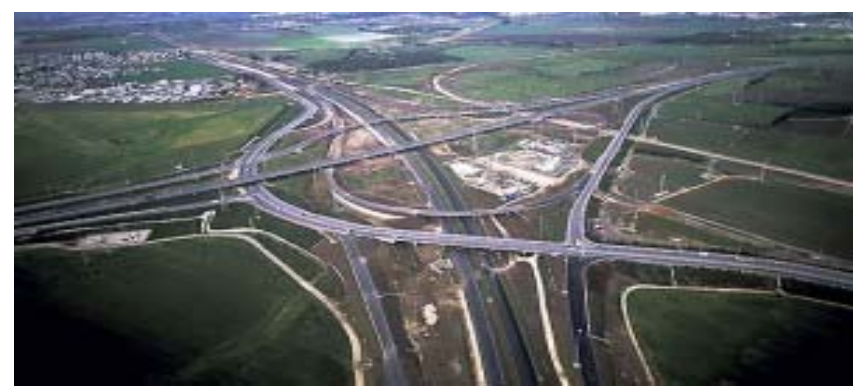

Fig. 2. Israel Highway

Hefer intersection along the old coastal road of Route 4 in Israel (Fig 2) was the place where a piezoelectric generator was put to the test and generated some 2,000 watt-hours of electricity. The setup consists of a ten-meter strip of asphalt, with generators lying underneath, and batteries in the road's proximity. The technology is based on piezoelectric materials that enable the conversion of mechanical energy exerted by the weight of passing vehicles into electrical energy. As far as the drivers are concerned, the road is the same.

Through the Israel's results, maybe we'll see these piezoelectric generators implemented worldwide in a few 


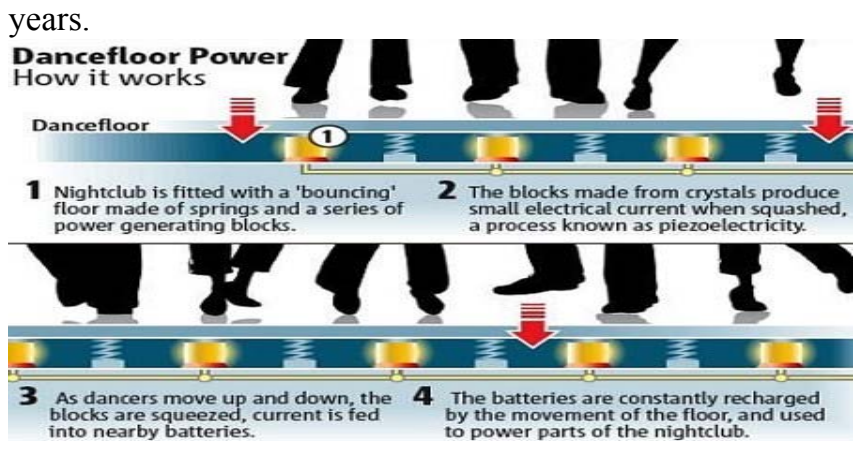

Fig. 3. Dance floor project in London

A famous nightclub in London exploited the principle of piezoelectricity in making its dance floor. The dance floor uses piezoelectricity where crystal and ceramics create a charge to generate electricity. The nightclub has a "bouncing" floor made of springs and a series of power generating blocks which produce a small electrical current when squashed. As dancers move the floor up and down to squeeze the blocks, the current is fed into nearby batteries which are constantly recharged by the movement of the floor. The electricity created in this way is used to power parts of the nightclub such as the sound and lighting. Fig 3 indicates how the dance floor project works.

This technology has many applications for the generation of electricity from the movement of people including pedestrian traffic in urban spaces, to vehicular traffic on roads.

\section{COMPONENTS OF DESIGN}

Piezo Ignitor

LM386 Audio amplifier

ATMEGA168 microcontroller

Pyroelectric crystal

Sound sensor

Battery

\section{A. Piezo Ignitor}

Piezo ignition is a type of ignition that is used in portable camping stoves, gas grills and some lighters. It consists of a small, spring-loaded hammer which, when a button is pressed, hits a crystal of PZT or quartz crystal [9]. Quartz is piezoelectric, which means that it creates a voltage when deformed. The volume of the ceramic element and the amount of stress exerted on the element are key factors in converting mechanical input to electrical energy. In squeeze-type piezoelectric fuel ignitors a static mechanical energy input - very low frequency, relative to the resonance frequency of the ceramic generates the electrical energy for ignition. In the impact ignition design a spring-loaded hammer delivers a dynamic input to the ceramic element. The pressure wave generated when the hammer strikes the element once is reflected multiple times in both the element and the hammer, in accord with the elastic and acoustical properties of the ceramic and the hammer. Until the flashover at the spark gap, stress varies along the height of the ceramic element, and exact values for voltage must be calculated by integration over the height of the element.
Note: Static Compressive Strength $(\mathrm{kg} / \mathrm{cm})>3000$ for all standard ignitor elements

Force / Voltage: g33 * (force $(\mathrm{N}) *$ thickness of ceramic (m)) /surface area of ceramic $(\mathrm{m} 2)=$ Volts

\section{B. ATMEGA168 Microcontroller}

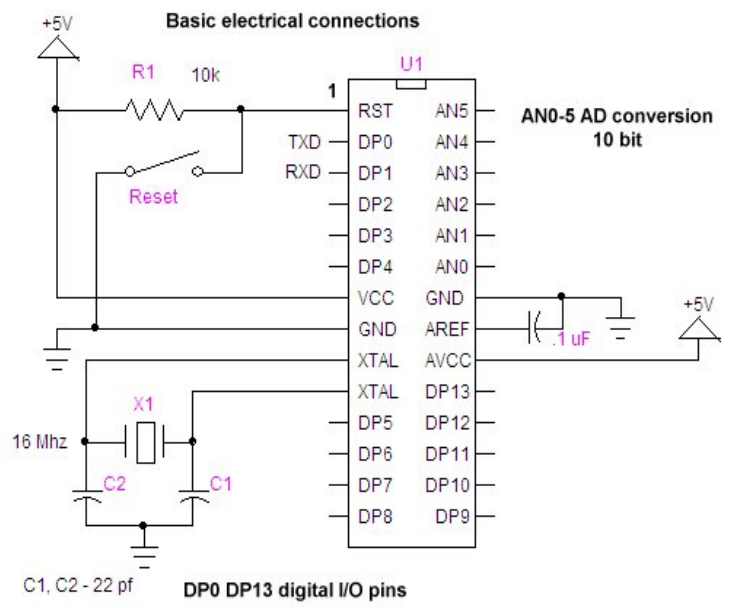

Fig 4 Atmega168 pin diagram

Fig 4 shows the pin diagram of an Atmega168 microcontroller

Atmega168/Arduino features:

14k flash RAM program storage

1024 bytes of static RAM for program memory

512 bytes of EEPROM

6 PWM outputs

6 10-bit A/D inputs

UART and SPI interfaces (SPI in not implemented!) 2

Hardware interrupts [6]

\section{Audio Amplifier}

The LM386 is a power amplifier designed for use in low voltage consumer applications. The LM386 pin diagram is shown in Fig 5. The gain is internally set to 20 to keep external part count low, but the addition of an external resistor and capacitor between pins 1 and 8 will increase the gain to any value from 20 to 200 . The inputs are ground referenced while the output automatically biases to one-half the supply voltage. The quiescent power drain is only 24 milliwatts when operating from a 6 volt supply, making the LM386 ideal for battery operation.

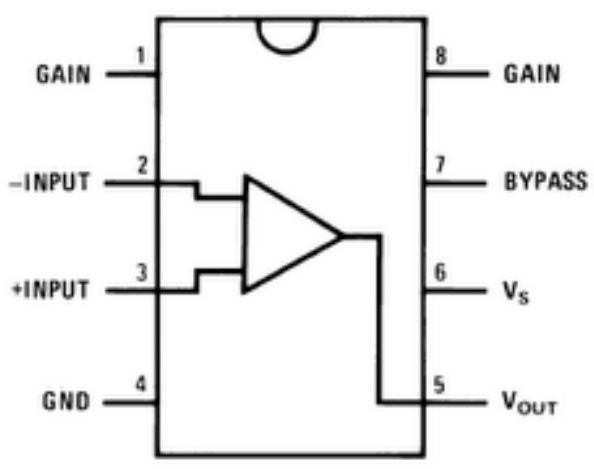

Fig 5 LM386 Audio Amplifier 


\section{Features}

- Wide supply voltage range: $4 \mathrm{~V}-12 \mathrm{~V}$ or $5 \mathrm{~V}-18 \mathrm{~V}$

- Low quiescent current drain: $4 \mathrm{~mA}$

- Voltage gains from 20 to 200

- Low distortion: $0.2 \%(\mathrm{AV}=20, \mathrm{VS}=6 \mathrm{~V}, \mathrm{RL}=8 \mathrm{Ohm}$, $\mathrm{PO}=125 \mathrm{~mW}, \mathrm{f}=1 \mathrm{kHz})$

The piezoelectric crystal bends in different ways at different frequencies and this bending is called the vibration mode. An important group of piezoelectric materials are ceramics.

The tabulation (Fig 6) below shows the vibration modes and the frequencies over which they can work.

\begin{tabular}{|l|l|l|l|l|l|l|l|l|l|}
\hline Vibration Mode & & \multicolumn{5}{|c|}{ Fequency (Hz) } \\
\hline $\begin{array}{l}\text { Flexure } \\
\text { Vibration }\end{array}$
\end{tabular}

Fig. 6 Vibration modes

\section{E. Pyroelectric Crystal}

Typical examples of pyroelectric crystals are tourmaline, lithium sulphate and monohydrate. Pyroelectric crystals can be regarded as having a built-in or permanent electric polarization. When the crystal is held at constant temperature, this polarization does not manifest itself because it is compensated by free charge carriers that have reached the surface of the crystal by conduction through the crystal and from the surroundings [8],[3] . However, when the temperature of the crystal is raised or lowered, the permanent polarization changes, and this change manifests itself as pyroelectricity [1]. The magnitude of the pyroelectric effect depends upon whether the thermal expansion of the crystal is prevented by clamping or whether the crystal is mechanically unconstrained. In the clamped crystal, the primary pyroelectric effect is observed, whereas in the free crystal, a secondary pyroelectric effect is superposed upon the primary effect. The secondary effect may be regarded as the piezoelectric polarization arising from thermal expansion, and is generally much larger than the primary effect [5],[7].

\section{F. Sound Sensor}

The Sound sensor module is a simple microphone. Based on the power amplifier LM386 and the electret microphone, it can be used to detect the sound strength of the environment. The value of output can be adjusted by the potentiometer. It has a wide supply voltage range of $4 \mathrm{~V}-12 \mathrm{~V}$, a maximum current supply of $8 \mathrm{~mA}$, low quiescent current drain of $4 \mathrm{~mA}$ and it is gain adjustable.

This module uses the LM386 power amplifier to strengthen the electronic signal produced by the electret microphone. When powered on, the SIG pin will output the signal regulated by LM386. The potentiometer at the output can be used to regulate the gain. The program below shows how to use the sound sensor to control the led [6]. Connect the Sound sensor to analog port A0 and the LED to port 12.The potentiometer is used to regulate the gain of the output signal. The larger the potentiometer, the larger the output signal. If the sound of the environment is bigger than the threshold, then the Led will be turned on.[4]

const int ledPin $=12$; / the number of the LED pin

const int thresholdvalue $=400 ; / /$ The threshold to turn the led on

void $\operatorname{setup}()$

\{

pinMode(ledPin, OUTPUT);

\}

Void loop()

\{

int sensorValue $=$ analogRead(A0);/use A0 to read the electrical signal

if(sensorValue $>$ thresholdvalue)

digitalWrite(ledPin,HIGH);//if the value read from A0 is larger than 400, then light the LED

$\operatorname{delay}(200)$;

digitalWrite(ledPin,LOW);

\}

\section{G. Battery}

One end of the circuit is connected to a battery. The battery is charged completely so that it can recycle the electrical power by converting it into mechanical energy and vice versa, so that it can be used in case of power failure power failure and in situations where reduction in electricity(by switching off the supply) expenditures is needed. The entire process mentioned in the design occurs only after the battery is fully charged. When the battery is completely charged, the supply gets cut off automatically from the device and finally the device operates by the use of battery.

\section{WORKING MODEL}

The block diagram of our model is shown in Fig 7.The mechanical energy produced by the device which is to be operated is given to the piezo ignitor. The piezo ignitor converts the given mechanical input to electrical energy [9].

1. One part of the electrical energy produced is given to the battery for charging.

2. The other part of the electrical energy is given to the 
LM386 audio amplifier by the voltage divider.

3. The heat generated by the ignitor is sent over to the pyroelectric crystal where it is converted into voltage [1], [2].

4. When the voltage produced is in the range of acceptable operating limit of the microcontroller, it is directly given to the controller.

5. If the heat generated is more than the critical value in the pyroelectric crystal, then a voltage divider is used to reduce the voltage and send the necessary voltage which the microcontroller can operate without any problem. The other part of the voltage which is divided by the voltage divider is given in parallel connection to the voltage given out from the ignitor crystal .The added voltage is given to the battery

6 . If the voltage coming from the pyroelectric crystal is below the critical value, then a part of the voltage coming from the piezo ignitor is added with the voltage coming from the pyroelectric crystal and is given to the microcontroller.

7. The noise signal in the amplifier is amplified to a particular gain value and sent to the sound sensor. The gain of the audio amplifier must be between 20 to 200 .

8. If the gain is too high and if it exceeds a critical value, it would damage the entire ignitor. So the sound sensor senses the level of gain and sends it to the microcontroller.

9. The microcontroller adjusts the gain of the amplifier accordingly that it does not cause damage to the ignitor. Proper input to the sound amplifier comes from the microcontroller after necessary programming.

10. Then the final value is given to the piezo ignitor.

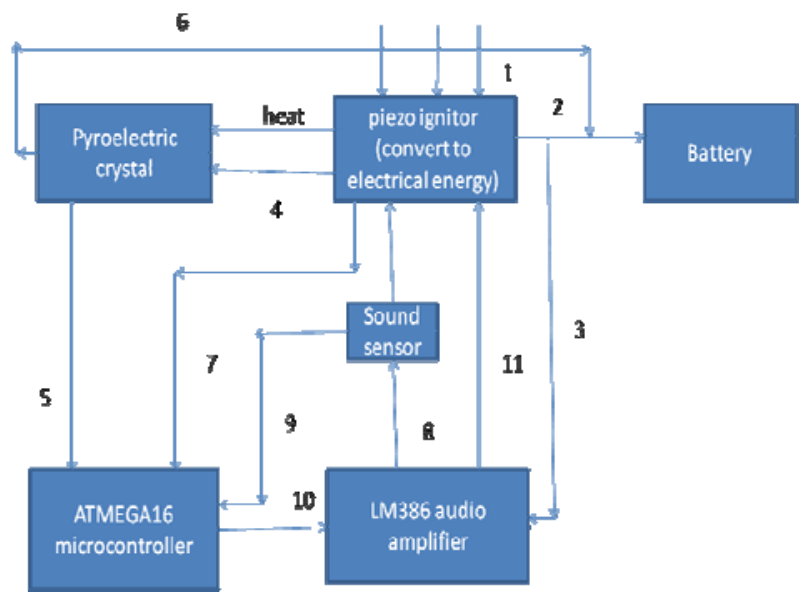

Fig. 7 working of the model

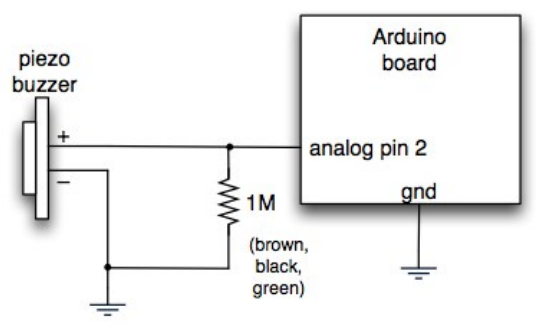

Fig 8 Microcontroller design

The coding [6] and the image (Fig 8) for increasing or decreasing the gain of audio amplifier are given below:-

void $\operatorname{setup}()$
\{

Serial.begin(9600);

pinMode(2,INPUT);

digitalWrite(2,HIGH);

\}

void loop()

\{

int $\mathrm{t}=0$;

float $\mathrm{x}$;

$\mathrm{x}=\operatorname{analog} \operatorname{Read}(2)$;

Serial.println(9-(x*.00878));

$\mathrm{t}=9-(\mathrm{x} * .00878)$;

delay $(1000)$;

while $(\mathrm{t}<5)$

\{

$\mathrm{t}=\mathrm{t} * 2$

\}

if $(\mathrm{t}>9)$

\{

$\mathrm{t}=9$;

\}

serial.print(t);

\}

\}

In the program above 9 is the maximum allowed value and 5 is the minimum allowed value, if the output drops below or above these values it is automatically detected by the controller and gain is adjusted.

All these process in the design occurs only after the battery is fully charged. When the battery is completely charged, the supply gets cut off automatically from the device and finally the device operates by the use of battery.

\section{Results}

From the analysis done so far these are the results(indicated by Table 1) got when a constant force is applied across a piezo electric crystal with $20 \mathrm{~cm}$ diameter:-

\begin{tabular}{|c|l|l|}
\hline \multicolumn{2}{|c|}{ TABLE I: RESULT TABLE } \\
\hline SNO & TYPE & $\begin{array}{l}\text { VOLTAGE (In } \\
\text { Volts) }\end{array}$ \\
\hline 1. & Single crystal & 0.67 \\
\hline 2. & $\begin{array}{l}\text { Parallel combination } \\
\text { (5 piezo electric crystals connected in parallel) }\end{array}$ & 2.28 \\
\hline 3. & $\begin{array}{l}\text { Series combination (5 piezo electric crystals } \\
\text { connected in series })\end{array}$ & 2.62 \\
\hline
\end{tabular}

\section{AdVAntages}

- This method gives rise to efficient recycling of energy.

- There is no loss in energy

- It can be used in situations in case of power failure or any emergency conditions

- This method reduces electricity expenditures. 
- This method of recycling can be used in many home appliances such as fans etc, it is also used in devices which produce noise and vibration while they are operating.

\section{REFERENCES}

[1] Carlioz.L "Temperature threshold tuning of a thermal harvesting switch" IEEE Trans on Solid State Devices pp-1385-1388, 2009

[2] Kukhtarev "Pyroelectric and photo-galvanic effects for generation of the focused electron beam, X-rays, photo-actuation and electric micro-propulsion", IEEE Trans pp-479-482, 2005

[3] Sidney Lang, "Pyroelectricity: From Ancient Curiosity to Modern Imaging Tool", Physics Today, August, 2005, pp. 31-36, and Sidney B. Lang, "Sourcebook of Pyroelectricity", (London: Gordon \& Breach, 1974)

[4] De Lima J.A. "A low-voltage programmable-gain CMOS amplifier with very-low temperature-drift" IEEE Trans and Circuits and Systems, pp-627-630 vol.I, 2001

[5] Jeffrey A. Geuther, Yaron Danon, "Pyroelectric Electron Acceleration: Improvements and Future Applications", ANS Winter Meeting Washington, D.C, November $14-18,2004$

[6] Richard H. Barnett, Sarah A. Cox, Larry D. O'Cull - Embedded C Programming and the Atmel AVR, Thomson Delmar Learning, 2002.

[7] W. Tornow, S. M. Lynam, and S. M. Shafroth, "Substantial increase in acceleration potential of pyroelectric crystals", Journal of Applied Physics, Vol. 107, 063302 (2010)

[8] Olsen, Randall B.; Evans, Diane " Pyroelectric energy conversion: Hysteresis loss and temperature sensitivity of a ferroelectric material". Journal of Applied Physics 54: 59411983

[9] George W. Taylor," Piezoelectricity “1985 46-61

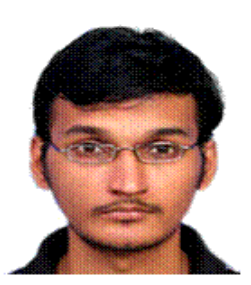

Arjun Agaram Mangad is currently pursuing his B.E (2008-2012) in ECE (Electronics and Communication Eng) at Sri Venkateswara College of Engineering in affiliation with Anna University.

He has undergone internship in Nokia pvt ltd, Sriperumbudur and NCR, Puducherry .His research interest are in the field of embedded systems. He has also attended project internship program in embedded systems and robotics in Simple Labs, Chennai. He is currently exploring other fields with an Ericsson empowerment program in the field of communication and an online course on Artificial intelligence conducted by Stanford University.

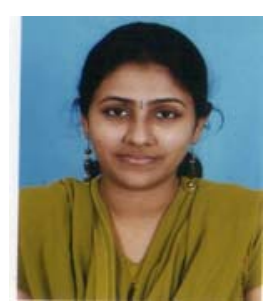

Sandhya Thiyagarajan is currently pursuing her B.E (Bachelor of Engineering) (2008-2012) in EEE (Electrical and Electronics Engg) at Meenakshi Sundararajan Engineering College in affiliation with Anna University.

She has undergone in plant training at Lucas Indian Service. Her research areas are computer networks and embedded systems .She did her research project with Cisco System Inc at San Jose, USA in the field of computer networks. She has attended project internship program in embedded systems and robotics. She has also presented many papers in National Level Technical Symposia. She is currently working on a research paper in car security system involving a GSM integrated embedded system..

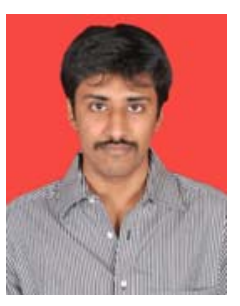

Ajay Sampath is currently pursuing his B.E (Bachelor of Engineering) (2008-2012) in Electronics and Communication Engineering (ECE) at Sri Venkateswara College of Engineering in affiliation with Anna University.

$\mathrm{He}$ has undergone in plant training at BSNL Chennai International Air Port. He has also done his internship at Reliance Communications. His research interests are in the area of embedded systems.He has also attended a project internship program in embedded systems and robotics. He has presented many papers in National Level Technical Symposia. He is currently working on a research paper in car security system involving GSM integrated in an embedded system.

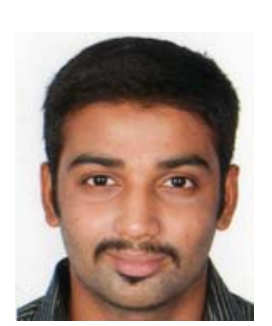

V.Arvind is currently pursuing his B.E (Bachelor of Engineering) (2008-2012) in Electronics and Communication Engineering (ECE) at Sri Venkateswara College of Engineering in affiliation with Anna University .

$\mathrm{He}$ has undergone in plant training at Doordarshan Kendra, Bsnl, Chennai International Airport And Nokia. His research interests are in the area of embedded systems and image processing .He has presented many papers in National Level Technical Symposia. He is currently working on a research paper in iris recognition systems involving the physiology behavior of iris surface deformation for various irises. 